\title{
Impresionar el punto ciego de la formación inicial de maestros
}

\author{
Impressing the \\ Blind Spot of Initial \\ Teacher Training
}

\author{
Impressionar o \\ ponto cego da \\ formação inicial de \\ professores
}

Ruth Milena Páez Martínez*

Fecha de recepción: 16-01-2017

Fecha de aprobación: 07-07-2017

\section{PARA CITAR ESTE ARTÍCULO}

Páez, R. (2018). Impresionar el punto ciego de la formación inicial de maestros. Nodos y Nudos, 42, 73-83.
* Maestra investigadora de la Universidad de La Salle. Licenciada en Educación Básica Primaria y magister en Educación por la Pontificia Universidad Javeriana de Bogotá. Doctora en Educación de la Universidad Pedagógica Nacional de Colombia. 


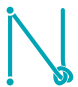

Volumen $5 \mathrm{~N} .{ }^{\circ} 42$ enero - junio de 2017 ISSN: 0122-4328 pp. 73-83

\section{RESUMEN}

Los maestros en formación requieren exponerse a situaciones que estimulen sus sentidos en escenarios o realidades directas, impresionar el punto ciego de su experiencia en contexto, antes de iniciar la formalidad de su práctica. Este ensayo se deriva de la reflexión suscitada por un texto que narra una experiencia de formación desarrollada a través de videofilmaciones de situaciones pedagógicas de docentes. A partir de alli, la autora recrea y plantea dos propuestas para la formación inicial de los maestros, una de inmediación y otra de mediación, ambas localizadas en la experiencia.

Palabras clave: Formación docente; experiencia; mediación; inmediación

\section{ABSTRACT}

Pre-service teachers require being exposed to situations that stimulate their senses in direct scenarios or realities, thus impressing the blind spot of their experience in a context before formally starting their practice. This essay is the result of a reflection about a text that narrates a training experience developed through video recordings of pedagogical situations of teachers. Based on this, the author recreates and presents two proposals for initial teacher training, one for immediacy, and another one for mediation, both found in experience.

Keywords: Teacher training; experience; mediation; immediacy

\section{RESUMO}

Os professores em formação precisam se expor a situações que estimulam seus sentidos em cenários ou realidades diretas, impressionar o ponto cego da sua experiência em contexto, antes de iniciar a formalidade de sua prática. Este ensaio deriva-se da reflexão suscitada por um texto que narra uma experiência de formação desenvolvida através de vídeos de situações pedagógicas de professores. A partir disso, a autora recria e expõe duas propostas para a formação inicial dos professores, uma de imediação e outra de mediação, as duas situadas na experiência.

Palavras-chave: Formação docente; experiência; mediação; imediação 
Antes de que los maestros en formación se enfrenten a la realidad de la práctica, conviene exponerlos a condiciones preparatorias, estimular sus sentidos en escenarios o realidades directas, impresionar el punto ciego de su experiencia en contexto. Este ensayo se deriva de algunos aportes de los docentes canadienses Michel Carbonneau y Jean-Claude Hétu (2005) a partir de una estrategia de videofilmaciones de situaciones pedagógicas y de su posterior análisis con estudiantes que se preparaban para ser maestros, en particular, de una idea apenas esbozada alli: "impresionar el punto ciego", y de mi propia experiencia inicial como docente de niños y niñas ${ }^{1}$. La primera parte recoge en breve el contexto de tal estrategia; la segunda, presenta un par de posibilidades en dicha formación desde mi propia experiencia, una de inmediación y otra de mediación. Me ubico en una especie de descripción fenomenológica, por fuera del rigor de este método.

Los profesores Carbonneau y Hétu de la Universidad de Montreal llevaron a cabo videofilmaciones de situaciones pedagógicas (registrando las clases de una maestra de primaria) con el fin de analizar el acto pedagógico y desde alli "extraer el saber vivencial del maestro" (2005, p. 107). En la medida en que las realizaban, se les ocurrió hacer un curso con los docentes en formación, a partir de los videos, y esto los llevó a reflexionar sobre la experiencia de formación en la práctica docente y a plantear de modo tentativo un modelo y unos principios de dicha formación.

Dentro de las razones que animaron tal decisión estuvo que, durante ese curso, advirtieron varios inconvenientes por parte de los docentes en formación: dificultad para reconocer las secuencias de la clase dentro de una continuidad y de un todo; dificultad para reconocer la interacción de la maestra observada con sus alumnos y deplorar su actitud en sus relaciones con estos, reprochar su falta de atención a las demandas de todos los estudiantes, calificarla de "muy severa", aun cuando se trataba de una maestra con buenas prácticas. Los investigadores cruzaron estas dificultades con las que tienen los maestros principiantes, dos de las cuales expusieron del siguiente modo:

1 El texto fue leído en el seminario de docentes de la Maestría en Docencia de la Universidad de La Salle de Bogotá en el segundo semestre de 2015, a propósito del libro La formación profesional del maestro. Estrategias y competencias (2005). a. Los docentes nóveles se sorprenden y desconciertan al obtener poca respuesta de sus alumnos, aun cuando hayan planificado muy bien su clase. Una respuesta negativa al aprendizaje o de rechazo a las actividades propuestas por el docente, como si estuviesen "hablando idiomas diferentes"; o ante su disciplina (alumnos que se observan "desatentos" a la clase e irrumpen las actividades previstas). Una de las razones de esa poca respuesta de los alumnos es que estos docentes leen las situaciones de clase en su "instantaneidad", no alcanzan a tomar distancia en el momento que ocurren

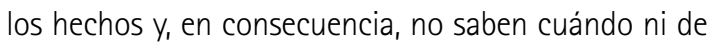
qué manera se requiere hacer un giro o un cambio al plan previsto.

b. Creen que se necesita seccionar la realidad para comprenderla. El maestro novel necesita tomarse un tiempo para analizar una situación y reaccionar ante ella en el mismo momento que se presenta. Su "formación teórica" le ha llevado a priorizar unos esquemas de análisis pedagógicos, más que unos esquemas de acción pedagógica, lo que se refleja en una lectura fragmentada de la situación. De ahí que supongan dos cosas: que las intervenciones de calidad siempre deben ser reflexivas y que el dominio de las teorias es la clave de una intervención exitosa; y que los aprendizajes prácticos pueden ser aislados como una reserva de saberes hasta el momento que se vuelvan a necesitar. Ahora, ambos esquemas son importantes solo que parecen no armonizados al interior de los programas universitarios de formación docente; esto se observa en la manera como los docentes principiantes tienden a resolver las situaciones pedagógicas que se les presentan. (Carbonneau y Hétu, 2005, pp. 126-138).

A partir de esto, los autores de la investigación proponen tres rasgos que debian contemplarse en la formación inicial de maestros² (2005):

a. Reconocer que la exposición a videos con situaciones reales de clase favorece una proyección de los futuros docentes, quienes "se ven" en las acciones de la maestra protagonista, una "acción

2 Estos investigadores también recogieron unos principios derivados de su experiencia, que ellos describen en un modo propositivo cuando se piensa en la formación inicial de los docentes (2005, pp. 129-132): a) aumentar el ritmo de la alternancia entre la formación para el análisis conceptual y la formación para la intervención (con la microenseñanza, los estudios de casos, la intervención ante grupos de niños, etc.); b) multiplicar las situaciones y los 'modelos' pedagógicos; c) valorar la toma de conciencia del estudiante sobre sus propios esquemas de acción; d) identificar marcos conceptuales flexibles que respeten esos esquemas de acción; e) no proponer un modelo sino a la persona como eje, el caso de una persona; f) desconfiar de las trampas de la práctica reflexiva. 
anticipatoria" de futuras situaciones escolares con las que se pueden enfrentar, que contribuye a la construcción de su primera identidad profesional o de su hipotético naciente "pensamiento profesional".

b. Reconocer que "el maestro, gracias a la experiencia, va constituyéndose su propia reserva de intervenciones. Cada situación exige una intervención particular $y_{1}$ en cuanto se presente la ocasión, el maestro podrá justificar la razón de su actuación".

c. Estimar que hay unos principios que guian la intervención docente, unos esquemas mentales que son la fusión de ideas, valores, conocimientos y experiencias.

Estas consideraciones responderian, al menos en parte, preguntas como: ¿Por qué el maestro actúa de uno $u$ otro modo ante una situación pedagógica determinada? ¿A qué fuentes, recursos o ideas acude para resolver o enfrentar las situaciones pedagógicas justo en el momento que se presentan (su lectura del acontecimiento, los principios derivados de teorías, su experiencia, su edad, sus ideologías, todo esto junto)?

Esto nos lleva a reparar en lo siguiente. Prepararse para la profesión docente desde fuera del ejercicio de esta, fuera del campo, fuera de la exposición a la práctica y a la experiencia parece ser una contradicción, pero también un error. La práctica es constitutiva de la docencia. Concentrar la formación de docentes o de futuros docentes en la comprensión y el análisis teóricos de una intervención, o de un caso, como algo exclusivo, mantiene la idea de una realidad fragmentada y superficial.

A cambio, mejor sería alternar en mayor medida la formación para el análisis conceptual y la formación para la intervención (con la microenseñanza, los estudios de casos, la intervención ante grupos de niños, etcétera).Poner a los estudiantes en contacto con "el carácter relativo de las modas pedagógicas" y comprometerlos para que construyan su propia identidad profesional. Valorar la toma de conciencia del estudiante sobre sus propios esquemas de acción. Identificar marcos conceptuales múltiples y flexibles que respeten esos diversos esquemas de acción. No proponer un modelo sino a la persona o a las personas con sus particulares modelos de intervención y saberes. $Y$ desconfiar de las trampas de la práctica reflexiva, es decir, aquellas que hacen creer que toda situación en la vida escolar debe pasar por el análisis reflexivo o que, en cualquier circunstancia, este debe ser el centro de la acción. Suponer esto puede "generar una comprensión fragmentaria de la realidad en detrimento de la capacidad de actuación". La práctica reflexiva sí es importante, pero se hace necesario dosificar su énfasis (Carbonneau y Hétu, 2005, pp. 129-132).

Desde una perspectiva antropológica sería interesante considerar o recordar, por ejemplo, que el ser humano no se constituye únicamente de lo racional y lo lógico, propios de un régimen de la vigilia y del progreso, sino también por la dimensión relacional y afectiva propia de un régimen del descanso y la ensoñación (Páez, 2017). En otras palabras, no es posible que un maestro esté en permanente alerta y reflexión. Las impresiones que experimenta en un aula y en el contexto donde esta se ubica también pueden convocarle al sueño, la ensoñación, la imaginación: a veces expresadas en el arte a través de relatos, ya sean fantásticos o míticos (Páez, 2013; Rodríguez, 2014), o relatos de vida propios o generados en sus estudiantes (González, 2016); a veces propiciados por el territorio natural donde se encuentran algunas escuelas, como el caso de las zonas rurales (González, Rodríguez y Viasús, 2016), entre otros.

En otras palabras, y guiando la reflexión hacia donde queremos, mejor sería estimular ese punto ciego ${ }^{3}$ de la experiencia que recibe miles de impresiones, sin la conciencia de su captura pero que, en efecto, son asimiladas por quien las percibe. Sabemos que el ojo humano tiene un punto ciego debido a que existe una zona de la retina en la que no hay células sensibles a la luz. La retina conecta los nervios con el cerebro a través de este punto ciego, que siempre está ante nosotros en un espacio que no vemos, un punto donde convergen los estímulos visuales que vienen del exterior y que luego acceden al córtex. Me resulta sorprendente. Hay ante nuestros ojos una parte del espacio, aunque no la veamos. ¡El origen de la visión

3 Tomo la imagen que traen Carbonneau y Hétu (2005) cuando pretenden explicar los mecanismos que entran en juego cuando el maestro en formación se expone a situaciones pedagógicas. 
es un punto ciego!, un punto medio o intermedio, carente de células fotosensibles, pero posibilitador de la conexión entre lo que se recibe de fuera y lo que llega al cerebro. Algo que capta todo lo que es posible captar y lo direcciona al interior sin que nos demos cuenta: percepción, sentidos, cerebro.

\section{Estimular situaciones de inmediación y mediación}

El estudio de las ingeniosas adaptaciones que ostentan

la anatomía, la fisiología y el comportamiento de los animales lleva a la conocida conclusión de que cada uno ha evolucionado de modo que se acomodara a la vida en el rincón que habitaba... cada animal vive además en un mundo subjetivo privado, no accesible a la observación directa. Este mundo se compone de información comunicada a la creatura desde fuera en forma de mensajes captados por sus órganos de los sentidos.

H. W. LISSMAN, EleCtric LOCATION BY FISHES, EN SCIENTIFIC AMERICAN

Así las cosas, parece ser que el acceso o la entrada al proceso de formación docente es más adecuado por vía experiencial y práctica. Ante esto, no se me ocurre otra cosa que pensar en lo que el antropólogo estadounidense Edward Hall planteaba en 1966, esa idea según la cual la experiencia personal se encuentre imbricada con la cultura de tal forma que las experiencias hondas, comunes y no declaradas que comparten los miembros de la misma se comunican sin saberlo. Para Hall, esto se sintetiza en el empleo que hace el ser humano del espacio: el que comparte con sus congéneres, pero también el que construye en torno suyo en el hogar, en la calle, en su sitio de trabajo, y que aumenta la identificación consigo mismo, intensifica la experiencia y disminuye la alienación (2011).

De acuerdo con esto, la experiencia que se tenga con el espacio podrá incrementar la relación con la cultura misma, la comprensión -no siempre expresa- de sus fluctuaciones y permanencias, a través de la relación con los otros que comparten ese espacio, de los objetos que permanecen o cambian en él; de las dinámicas entre las personas, y entre estas y los objetos en ese espacio.

¿Cuál es el espacio que comparte el maestro en formación con sus congéneres-alumnos y que podría aportarle a construir su identidad profesional? Parece ser que las facultades de educación han saturado a sus docentes en formación de "espacios virtuales" mediados por textos gráficos en sus diversos formatos 0 , en el mejor de los casos, por observaciones de clase o videos, pero que, en aras de priorizar las comodidades de la estructura administrativa que soporta los programas de licenciatura, han puesto al margen las posibilidades directas de que ellos tengan una experiencia con el espacio vital de una clase. Si se quiere construir relaciones con el espacio y con quienes lo habitan, mejor será que esta experiencia se dé en un espacio concreto y en un tiempo continuo y largo, no a pedazos (respondiendo al restringido horario de los programas). Esto invitaría a girar la perspectiva de la práctica pedagógica y, ojalá, a conseguir una inmersión de los docentes en formación en el espacio-tiempo del aula y del contexto escolar, una mayor presencia, aun cuando no sean docentes "titulares".

Vuelvo a Hall. Cuando se quiere comprender al ser humano "tenemos que saber algo de la naturaleza de sus sistemas de recepción y de cómo la información recibida de ellos se modifica por la cultura" (2011, p. 56). Dos alternativas encuentro, ambas de exposición a situaciones vivenciales, solo que una determinada por las inmediaciones y otra por las mediaciones.

\section{Exposición a situaciones de inmediación}

Edward Hall habla de "receptores de inmediación" para referirse a lo que está contiguo o pegado a nosotros, 0 sea lo relativo al tacto, a las sensaciones que recibimos de la piel, las mucosas, los músculos. Por ser el tacto un sistema tan antiguo como la misma vida, la capacidad de respuesta de la piel a los estímulos es un criterio vital. Cuando se trabaja con niños pequeños esto es más evidente, dado que la distancia entre el cuerpo de la maestra y sus niños es de tipo personal (hay una especie de esfera o burbuja protectora que se mantiene 
entre los cuerpos de unos y otros, entre 45 y $120 \mathrm{~cm}$, como la distancia entre pupitres o en una formación) e incluso íntimo (cuando la presencia de otra persona es inconfundible por la afluencia de datos sensoriales: la mano de la maestra que se pone sobre la del niño para marcar un ritmo musical o un forma gráfica, o para ubicarlo en un espacio, en una distancia de menos de $45 \mathrm{~cm}$ ), según la clasificación que asimila este antropólogo.

La experiencia en situaciones de inmediación corresponde al "mundo subjetivo privado", no es accesible a la observación directa de terceros, es única. Ni el sujeto mismo de esa experiencia alcanza a prever sus efectos ni posibilidades. Como cuando me inicié como maestra: tenía 17 años y los niños, 4. Mis opciones de estudio profesional se hallaban por otro camino, más con la idea de salir del grupo de familiares insertos en el campo escolar. Sin embargo, ingresé a trabajar en un colegio de preescolar para "no perder el tiempo" mientras iniciaba estudios en matemáticas. Pasadas varias semanas de mi labor, estos niños de kínder me encaminaron hacia un reconocimiento: ellos necesitaban de mi presencia, interacción y "transacción" permanente, no había otra persona que me supliera, yo era importante para ellos; pero además no tardé en darme cuenta de que yo también los necesitaba porque había creado un vínculo con ellos. Fue así como, a cambio de huir, estos pequeños me ayudaron a tomar la decisión más importante de mi carrera profesional: sería maestra de niños.

Resultó impactante para mí la necesidad de cercanía física y afectiva de estos niños. Algo pasaba en mi cuerpo semiadolescente, que de pronto se veía apretado por un "abrazo" a la cintura, o empujado en un juego de escondidas o acariciado por la curiosidad de tocar un accesorio o la textura de una prenda. No habia que pensar, en medio del inesperado roce; solo habia que responder a ese tacto infantil y aprender que era necesaria esta cercanía, alzarlos, subirlos, ayudarlos a vestir y a comer, tomar su mano para simular formas y movimientos o para guiar sus primeros trazos de color y de grafía, abrazar sus indefensos cuerpos ante un dolor o desaire, en fin. Sus diferentes aromas me hacian imaginar cómo serían sus casas y sus familias, que no conoci muy bien. Vienen a mi mente el aula, el patio de juego y los niños demandando de mí proximidad y relatos a lo largo de su jornada preescolar.

Tiempo más tarde, a propósito de un seminario de fenomenología, que vendría a ser una situación de mediación de aquella experiencia vivida, me pregunté ¿qué hace distinto el cuerpo de la maestra de preescolar? Esto intenté aclarar:

Lo diferente es la forma como, en su relación con los niños que han sido puestos a su cuidado y formación, este experimenta la necesidad de proteger, de ayudar y de guiar en un acercamiento corporal mutuo que contribuya a tales fines. Lo que pasa por el cuerpo de la docente va dejando en ella un aprendizaje, una marca que será referente para futuras interacciones. Los niños necesitan cercanía, presencia física, la corporalidad de la maestra para conocer; el cuerpo de la docente requiere lo mismo para enseñar [...] El cuerpo visible de la maestra recibe en su "seno" el cuerpo de los niños que están bajo su cuidado y formación. Es un cuerpo externo volcado en acción hacia otros cuerpos externos. Es un cuerpo expuesto y dispuesto al afuera; un cuerpo preparado (y que se prepara) para ocupar un espacio al lado de otros cuerpos que están formándose para tener un lugar también, su propio espacio... (Páez, 2008, pp. 127-128).

Resumo. Esta experiencia de inmediación no habría sido posible dentro del tiempo programado para la práctica de mi bachillerato pedagógico, ni siquiera la práctica por un día (me refiero a las horas destinadas para "ser practicante", a veces con la compañia de un docente que te observa). Eso sí, había quedado grabado en mi esquema mental de práctica la secuencia de una clase y su organización inductiva o deductiva que siempre fueron de ayuda. Pero ni con las teorias de Piaget y Vygotsky, ni con el Humano, demasiado humano de Nietzsche que debiamos leer y estudiar en grado undécimo habría logrado esa experiencia. Sus enunciados, aunque los supiera identificar, distaban de la realidad experiencial que tuve. Era necesario situarme en un espacio-tiempo real y, sin saberlo, dejar que el punto ciego de mi experiencia se impresionara corporal, afectiva y cognitivamente, y que esto empezara a ser parte de mi equipamiento como maestra.

Retomo a Hall. Al comienzo somos una "especie de contacto" y, aunque no solo seamos "de contacto", la recepción que logremos por este medio impresiona 
la vida. Esto aplicaría de modo particular para los docentes en formación que se inician trabajando con niños. Y voy a Duch y Chillón (2012): no sólo de logos está hecho el ser humano, también de mythos.

\section{Exposición a situaciones de mediación}

Dicen Lluis Duch y Albert Chillón (2012) que el ser humano comparte con todos sus congéneres la condición mediada y la condición de la comunicación, de ahí que cree extensiones o prolongaciones de sí a través de

...herramientas y convenciones, iconismos y escrituras, rituales y cultos, relojes y metros, espejos y leyes, dioses y demonios, memoria y esperanza, instituciones y tumbas. Si no dispusiera de mediaciones, el anthropos se hallaria hincado en su hábitat: incapaz de comprenderlo y transformarlo, arrastrado por un biológico existir que transcurriria sin tiempo, incapaz de configurar su propia historia y biografia. (p. 23).

Se está usando tanto el término mediaciones en el contexto educativo, que pareciera suficiente mencionarlo para saber de qué se habla. Hay varias definiciones, pero prefiero las que provienen de la antropología pues nos recuerdan que somos seres hechos de tiempo y de espacio, finitos y frágiles. Y en esta finitud y fragilidad ubico al maestro en formación para decir que necesita ser expuesto a mediaciones que activen sus sentidos, sus afectos y su intelecto como la narrativa, la pintura, el cine o el arte en general, mediaciones que alimenten su esperanza y la de otros.

Esta vez, oído y vista son los sentidos privilegiados. Luego del tacto se fortalecieron en su orden, el oído y la vista, "receptores a distancia". En efecto, la vista fue el último sentido desarrollado por el ser humano, más que la olfacción debido a que los ancestros dejaron el suelo y ganaron los árboles: "la visión esteroscópica es esencial en la vida arbórea. Sin ella sería muy arriesgado saltar de rama en rama" (Hall, 2011, p. 57). Gracias al desarrollo de la vista, el ser humano adquirió la capacidad de planear, de compilar datos más complejos y de favorecer el pensamiento abstracto. Adquirió también la capacidad de crear las artes: "la poesía, la pintura, la música, la escultura, la arquitectura, la dan$z a$, dependen principalmente, siquiera no de un modo exclusivo, de los ojos y los oídos" (p. 55).
En este sentido, aproximarse a la realidad del acto pedagógico bien puede nutrirse de mediaciones como estas. A mayor exposición de mediaciones, mayores posibilidades comprensivas del espacio-tiempo donde se encuentran el maestro y sus alumnos. Tales mediaciones pasarán también por el punto ciego de la experiencia, aun cuando no se ejerza en su momento la docencia. Si bien la experiencia no se puede transferir ni observar de modo directo, habrá indicios de ella en las manifestaciones de quien sí la tiene.

Una película como Todo empieza hoy de Bertrand Tavernier (1999)4 ${ }^{4}$, por ejemplo, tampoco podrá trasponer a sus espectadores la vivencia del director de una escuela y de sus docentes, pero si podría acercar a los nóveles maestros a conocer la fuerte influencia del contexto sobre la escuela, en particular en situaciones de desempleo, pobreza, desintegración familiar y negligencia gubernamental, y las variadas maneras como podria hacérsele frente a una situación así. Los docentes espectadores no son los protagonistas pero podrian identificarse con ellos, contemplar alternativas ante los problemas planteados en el filme $y$, en el futuro, cuando encaren realidades similares, podrian filtrarse soluciones similares o más adaptadas al contexto real donde se encuentren.

Cerrando. Se necesita que los maestros en formación se expongan a la realidad de la práctica en situaciones de inmediación y de mediación, sí. Impresionar los sentidos, las emociones y la mente son tareas primordiales en la formación de los docentes que no pueden reducirse a las horas de una programación.

En la inmediación,

...las acciones entre maestros-maestros, maestros-alumnos, alumnos-alumnos, y entre estos y los espacios con sus objetos, se asemeja a la de una presencia [...] Esta presencia se vuelca sobre el acto físico de estar en algún lugar concreto [...] La pregunta por el espacio es la pregunta por la presencia del otro. (Páez, 2016, p. 108).

4 Esta cinta narra la difícil cotidianidad de una escuela de preescolar con una población marginada en Hernaing (Francia), donde Daniel Lefebre, director de la misma, debe hacer frente a obstáculos burocráticos y a una crisis social, incluida la familia, todo por el bienestar de sus estudiantes. 


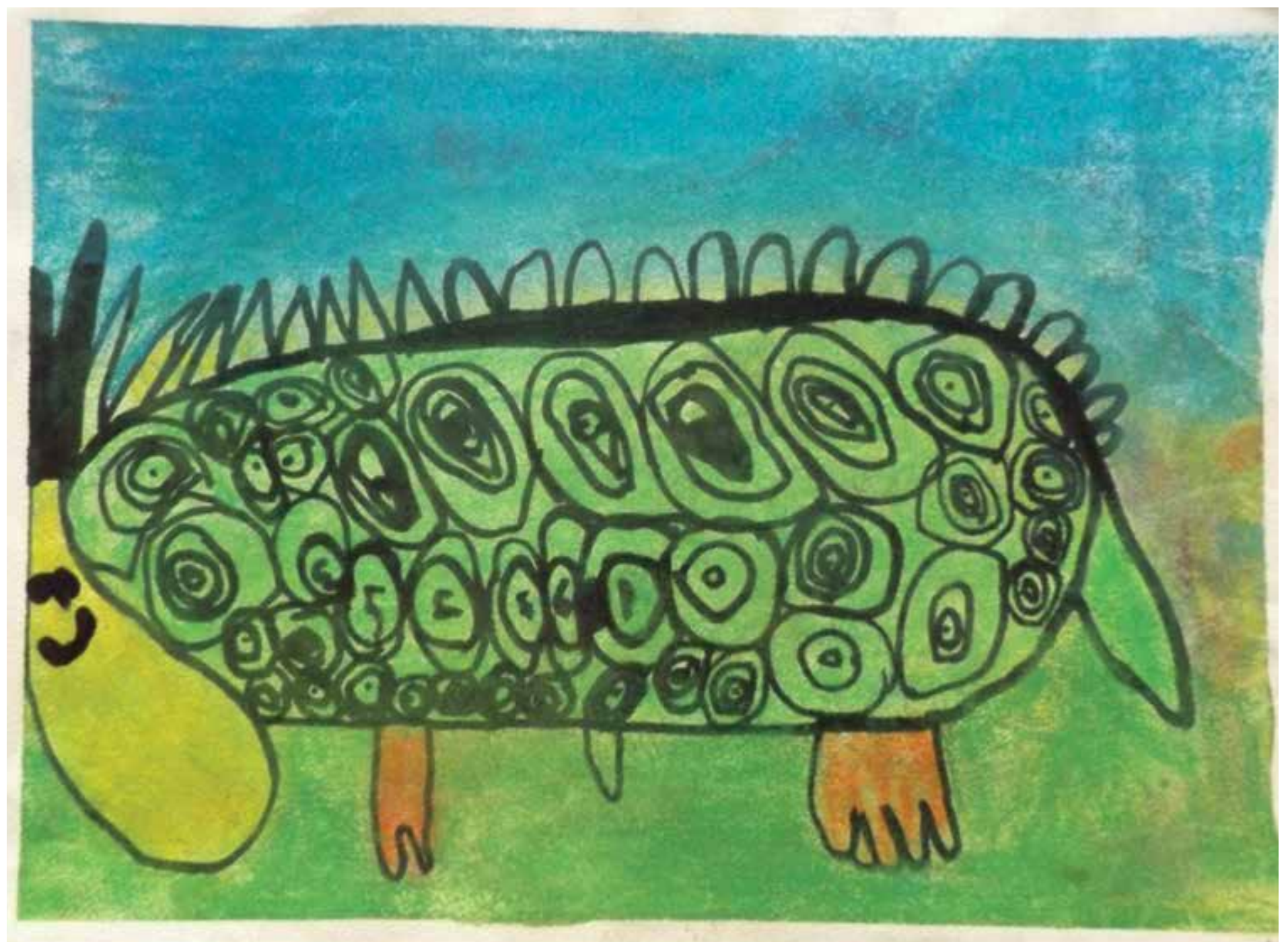

2
autor :
título :
año :
Técnica : 
Es una pregunta por el cuerpo. Acá, la reflexión puede ocurrir sin que se le prefigure o proyecte con antelación, pero también puede no ocurrir mientras el cuerpo se expone a las impresiones que captura por el tacto. Tales impresiones aportan en la formación del docente inicial en la medida en que la presencia presente le "recuerda" que él mismo es susceptible de impresionarse y de entrar en relación inmediata con unos niños o unos adolescentes que son sus estudiantes (aun cuando no sea del todo consciente de ello), y en cuanto le permiten gozar de la vitalidad de sus congéneres (muchas veces sin percatarse de ello).

En la mediación, las relaciones entre los docentes y los estudiantes los invitan a comunicarse en el sentido de encontrarse con el otro o la otra en la imagen (Páez, 2017), una imagen provocada por las múltiples posibilidades que ofrece el lenguaje en las narraciones y los ritos, por ejemplo.

La mediación contribuye en la formación del docente novel por la presencia ausente de símbolos que le recuerdan su historia personal en un movimiento hacia el pasado y hacia el futuro. Las impresiones del oído y la vista están mediadas por diversas construcciones culturales.

Exponerse e impresionarse son constitutivos de la experiencia formativa.

\section{Referencias}

Carbonneau, M. y Hétu, J. (2005). La formación práctica de los maestros y el nacimiento de una inteligencia profesional. En La formación profesional del maestro. Estrategias y competencias. México: Fondo de Cultura Económica.

Duch, Ll. y Chillón, A. (2012). Un ser de mediaciones. Antropología de la comunicación (vol. 1). Barcelona: Herder.

González, M. (2016). Narrar-nos es formar-nos: las historias de vida en la formación de maestros. Nodos y Nudos, 4(40), 103-116.

González, M. Rodríguez, S. y Viasús, K. (2016). Las experiencias pedagógicas de los educadores de Inzá, apuesta por una educación del campo. Nodos y Nudos, 5 (41), 7-20.

Hall, E. (2011). La dimensión oculta. México: Siglo XxI.

Páez, R. (2008). El cuerpo de la maestra de preescolar. Revista Iberoamericana de Educación, 47, 123-139.

Páez, R. (2013). Esbozo para una didáctica del símbolo en la educación básica. Infancias Imágenes, 12(1), 124-129.

Páez, R. (2016). Entre el espacio sígnico y simbólico: a propósito de la Guía de las escuelas. En F. Vásquez (Ed.), Relectura de la Guía de las escuelas: una mirada desde la educación superior (pp. 93-108). Bogotá: Ediciones Unisalle.

Páez, R. (2017). Lo mítico en la formación de los niños. Una propuesta para integrar lo imaginario, la narrativa, la comunicación, el rito, la memoria y lo estético en la escuela. Bogotá: Clacso.

Rodríguez, C. (2014). La literatura en la escuela: una mirada desde los textos, los contextos y el maestro. Nodos y Nudos, 4(36), 31-42.

Tavernier, B. (Dir.). (1999). Ça commence aujourd'hui. Francia: Les Films Alain Sarde/Little Bear/TF1 Films Production. Video-película. 


\section{DIÁLOGO DEL CONOCIMIENTO}

\section{La formación de maestros: entre la teoría y la práctica}

¿Cuál es la dosis adecuada de teoría y de práctica en la formación inicial de maestros? Desde el siglo xix empezó la controversia acerca de lo más pertinente para la formación de un buen maestro. Si el énfasis debe hacerse en dotarlo de herramientas teóricas, principalmente, o si estas deben equilibrarse con una dosis equivalente de pedagogia, es decir, de una especie de saber que contempla la práctica de alguna manera. Al abrirse el debate se producen, como efecto, diferentes tendencias y modelos pedagógicos, dependiendo de cómo quede ubicada alli la práctica. Se aprende a ser maestro en la práctica, de la misma manera que se aprende a ser cirujano en el quirófano.

Los desencuentros entre teoria y práctica hoy no cesan y se hallan a lo largo de dos siglos de propuestas y debates. Hoy podria decirse que la teoria es una herramienta que contribuye a la formación, al tiempo que no puede decirse que es la única. De hecho, la configuración de la teoría, para que se considere como tal, hubo de sacrificar aspectos de la realidad para poder ganar el dominio idóneo de un sector de esta.

Cuando se da el peso dominante y exclusivo a la teoria en un proceso formativo para ser maestro, es porque se "suponen dos cosas: que las intervenciones de calidad siempre deben ser reflexivas y que el dominio de las teorías es la clave de una intervención exitosa", dice la autora. Esto nos lleva a pensar que la segunda afirmación puede parecer consecuencia de la primera: a mayor reflexión, mayor calidad y el lugar más refinado, más elaborado, de la reflexión es la teoría. Sin embargo, se nos advierte que antes de llegar alli no solo hay que detenerse, sino que puede ser interesante dudar de la primera afirmación.

En la medida en que los aprendizajes prácticos son importantes en el ejercicio docente $-\mathrm{y}$ por tanto en la formación-, ¿dónde y cómo hacerlos coincidir con aprendizajes teóricos? Al respecto, en el texto encontramos que "ambos esquemas son importantes solo que parecen no armonizados al interior de los programas universitarios de formación docente". En efecto, no se armonizan alli y quizá nunca terminan de armonizarse en lugar alguno, entendiendo por armonía la claridad y precisión para ensamblarse uno con el otro. Posiblemente sean relaciones siempre conflictivas. 
Al igual que se afirma que "el maestro, gracias a la experiencia, va constituyéndose su propia reserva de intervenciones", podrá decirse que la teoría también es una buena reserva disponible para usarse cuando las circunstancias asi se lo exijan al maestro. Por cuanto la experiencia es su otra reserva, las dos dimensiones, teoría y práctica, se disputan permanentemente ser la guia de las acciones del ejercicio docente; o es una mezcla que se agita en el fuero interno del maestro. La autora se decide por esto último y proporciona a dicha mezcla la denominación de "esquemas mentales", en los cuales convergen "ideas, valores, conocimientos y experiencias".

El caso es que los "esquemas mentales" están hechos de ingredientes heterogéneos y aqui volvemos al punto inicial: ¿a cuál de estos apostarle con mayor certidumbre para llegar a la formación deseada? Las dos dimensiones de las que hemos venido hablando, teoría y práctica, son de constituciones diferentes. Mientras la teoría se deja acumular y ordenar más fácilmente (razón por la cual tenemos disciplinas), la experiencia parece menos dócil, más caótica. De este modo, lo que queda es aceptar que se aprende a ser maestro en la escuela, o que su formación es incompleta si no pasa por la escuela. Se han hecho muchos esfuerzos para atrapar más aspectos involucrados en el ejercicio docente, desde metodologias diversas (etnografia, estudios de caso, análisis de contenido, entre otros); miradas que aportan comprensión, pero que no sustituyen la práctica.

La sospecha, y propuesta, de la autora es ir más allá de iniciativas exclusivamente racionales, incluyendo en los procesos formativos aspectos como la imagen, los sentidos y lenguajes que trascienden las disciplinas. Es decir, prohijar ingredientes menos ortodoxos en la formación para que, incluso, la historia personal del maestro en formación tenga cabida.

RAÚl BARRANTES 